\title{
Study of neutron spectra in extended uranium target. New ex- perimental data
}

\author{
M. Paraipan ${ }^{1,2, \star}$ and E\& T Collaboration \\ ${ }_{1}^{1}$ Joint Institute for Nuclear Research,6, Joliot Curie St., 141980 Dubna, Moscow Region, Russia \\ ${ }^{2}$ Institute of Space Science,409, Atomistilor St., Măgurele - Bucharest, 077125, Romania
}

\begin{abstract}
The spatial distribution of neutron fluences in the extended uranium target ("Quinta" assembly) irradiated with $0.66 \mathrm{GeV}$ proton, $4 \mathrm{AGeV}$ deuteron and carbon beams was studied using the reactions with different threshold energy $\left(E_{t h}\right)$. The data sets were obtained with ${ }^{59} \mathrm{Co}$ samples. The accumulation rates for the following isotopes: ${ }^{60} \mathrm{Co}\left(E_{t h} 0 \mathrm{MeV}\right),{ }^{59} \mathrm{Fe}\left(E_{t h} 3 \mathrm{MeV}\right),{ }^{58} \mathrm{Co}\left(E_{t h} 10 \mathrm{MeV}\right),{ }^{57} \mathrm{Co}\left(E_{t h} 20 \mathrm{MeV}\right),{ }^{56} \mathrm{Co}\left(E_{t h}\right.$ $32 \mathrm{MeV}),{ }^{47} \mathrm{Sc}\left(E_{t h} 55 \mathrm{MeV}\right)$, and ${ }^{48} \mathrm{~V}\left(E_{t h} 70 \mathrm{MeV}\right)$ were measured with HPGe spectrometer. The experimental accumulation rates were compared with the predictions of the simulations with Geant 4 code. Substantial difference between the reconstructed and the simulated data for the hard part of the neutron spectrum was analyzed.
\end{abstract}

\section{Introduction}

The use of proton beams for energy production in accelerator-driven systems (ADS) was extensively investigated [1]-[3] and the general opinion is that the optimal energy of proton beam lies in the range 1-3 GeV. The possibility to use heavy-ion beams for ADS was less analyzed and the conclusions of authors are contradictory [4]-[5]. Simulations performed using the code Geant 4 in a quasi-infinite uranium target predict that one cannot improve the energetic efficiency of proton or deuteron beams by increasing the beam energy above $2-3 \mathrm{AGeV}$, but one can get a higher efficiency by accelerating heavier ions [6]. Based on this fact, a series of experiments with ion beams started at JINR. A more detailed analysis of particle distributions and spectra was realized by measuring the accumulation rates of isotopes with threshold energies from 0 to $70 \mathrm{MeV}$, using probes of ${ }^{59} \mathrm{Co}$ placed at different positions inside the target. In this study the distribution of the accumulation rates of these isotopes in the uranium target "Quinta" irradiated by $0.66 \mathrm{GeV}$ proton, $4 \mathrm{AGeV}$ deuteron and carbon beams is presented. The experimental data are compared with the predictions obtained using Geant4.10.1 [7] simulation code.

\section{Experimental setup and method}

"Quinta" is an extended ${ }^{n a t} U$ target composed from 5 section. Each section is an assembly of rods with diameter $36 \mathrm{~mm}$ and length $104 \mathrm{~mm}$ placed in a hexagonally shaped Al container. There are gaps of $17 \mathrm{~mm}$ between sections. In these gaps we introduce Al plates with probes from various

^e-mail: mparaipan@sunhe.jinr.ru 
materials, placed at different radial distances from the beam axis. The first section has a beam window with diameter $144 \mathrm{~mm}$. The entire assembly is surrounded with $10 \mathrm{~cm}$ thick $\mathrm{Pb}$ blanket with external dimensions $300 \mathrm{~mm} \times 300 \mathrm{~mm} \times 900 \mathrm{~mm}$ and beam window $150 \mathrm{~mm} \times 150 \mathrm{~mm} \times 100 \mathrm{~mm}$. The scheme of the target is presented in figure 1 . The longitudinal distances are measured from the surface of the blanket in the beam direction.

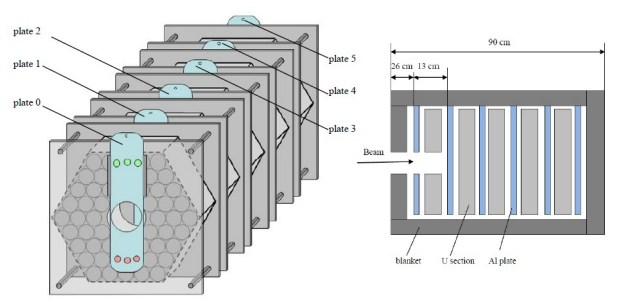

Figure 1. The scheme of "Quinta" setup.

Cobalt was chosen as material for the samples because is mono-isotopic $\left({ }^{59} \mathrm{Co}\right)$ and because there are sufficient experimental data on the excitation function under irradiation by neutrons and protons for the following isotopes covering a wide neutron energy range: ${ }^{60} \mathrm{Co}$ (threshold energy $E_{t h}=0$ $\mathrm{MeV}),{ }^{59} \mathrm{Fe}\left(E_{t h}=3 \mathrm{MeV}\right),{ }^{58} \mathrm{Co}\left(E_{t h}=10 \mathrm{MeV}\right),{ }^{57} \mathrm{Co}\left(E_{t h}=15 \mathrm{MeV}\right),{ }^{56} \mathrm{Co}\left(E_{t h}=25 \mathrm{MeV}\right),{ }^{47} \mathrm{Sc}$ $\left(E_{t h}=55 \mathrm{MeV}\right)$, and ${ }^{48} \mathrm{~V}\left(E_{t h}=70 \mathrm{MeV}\right)$. Available experimental data on an isotope cross section is an important condition for using the isotope for the comparison with the simulated spectra and for the reconstruction of the energy spectrum of the particles. For neutrons the experimental data on the excitation functions in Co are available for energies until $180 \mathrm{MeV}$. For higher energies we use the values obtained with Geant 4 and the binary cascade model (which provides the predictions closest to the experimental data for both protons and neutrons) properly adjusted to agree with the experimental data. An example of the simulated neutron spectrum registered at a longitudinal position of $39 \mathrm{~cm}$ and a radius of $8 \mathrm{~cm}$ is shown in figure 1a. This figure also shows the threshold energy for the isotopes. The probabilities of isotope production corresponding to the spectrum in figure 2a are shown in figure $2 b$, as functions of the neutron energy. This figure illustrates the energy interval in which the accumulation rate of a given isotope provides information on the neutron fluence. These energy intervals are approximately: $3 \mathrm{MeV}-40 \mathrm{MeV}$ for ${ }^{59} \mathrm{Fe}, 10 \mathrm{MeV}-40 \mathrm{MeV}$ for ${ }^{58} \mathrm{Co}, 20 \mathrm{MeV}-200$ $\mathrm{MeV}$ for ${ }^{57} \mathrm{Co}, 30 \mathrm{MeV}-300 \mathrm{MeV}$ for ${ }^{56} \mathrm{Co},>60 \mathrm{MeV}$ for ${ }^{47} \mathrm{Sc}$, and $>80 \mathrm{MeV}$ for ${ }^{48} \mathrm{~V}$.

The target was irradiated by protons with an energy of $0.66 \mathrm{GeV}$, deuteron and carbon beams with an energy of $4 \mathrm{AGeV}$. The beam position was monitored using the multiwire and pad ionization chambers. Solid state track detectors placed on the front side of the target were also used. The total beam intensity was measured with ionization chambers (for deuteron and carbon beams) and by activation of $\mathrm{Al}$ foil for the proton beam (reaction ${ }^{27} \mathrm{Al}(\mathrm{p}, \mathrm{x}){ }^{24} \mathrm{Na}$ ). Detailed description of the beam monitoring system can be found in [8]. The values of the integral beam intensity were $2.5 \cdot 10^{15}$ for 

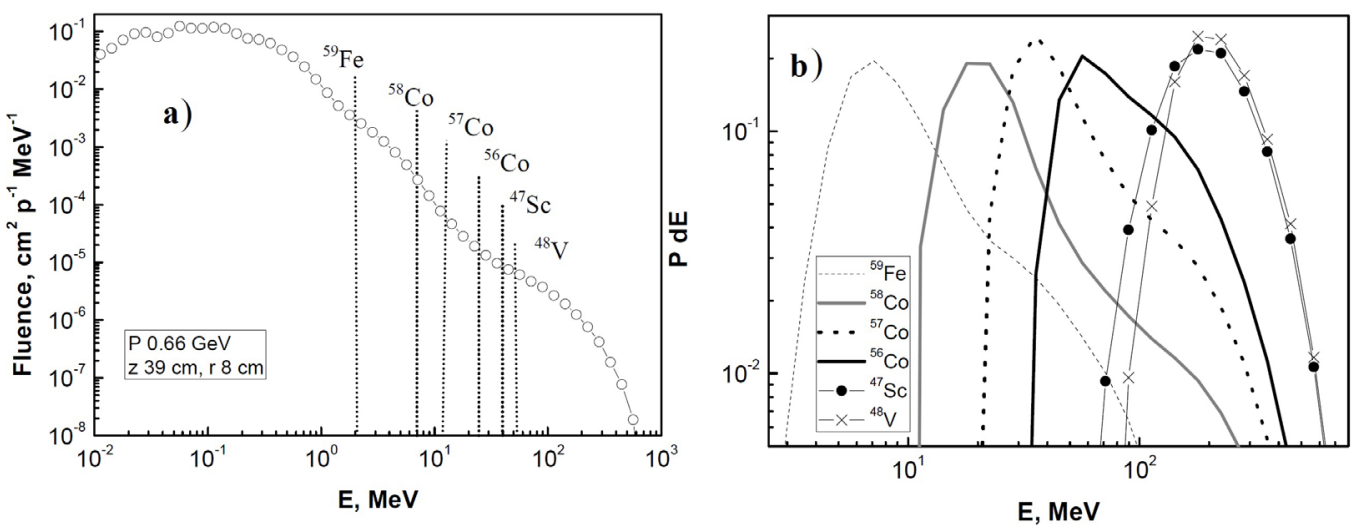

Figure 2. Simulated neutron spectrum at $\mathrm{z}=39 \mathrm{~cm}$ and $\mathrm{r}=8 \mathrm{~cm}$ (a); corresponding probabilities of isotope production (b).

protons $0.66 \mathrm{GeV}, 6.2 \cdot 10^{12}$ for deuteron $4 \mathrm{AGeV}$ beam, and $4.8 \cdot 10^{10}$ for carbon $4 \mathrm{AGeV}$ beam, measured with a relative error of $10 \%$. The accumulation rates of the isotopes in each sample were determined by measuring the $\gamma$-activities using the HPGe $\gamma$-spectrometers. The spectrometers were calibrated with a set of standard $\gamma$-sources. The reported error for the activity of $\gamma$-standards is $2 \%$.

The simulations with Geant 4 were performed with two combinations of models for the inelastic hadron interaction. In the variant labeled "BC-Bert" we used the Bertini cascade model for hadrons and Geant 4 binary cascade model for ions. The deexcitation stage of the interaction was described by the standard Geant 4 model for deexcitation. In the variant labeled "INCL" the Liege cascade model was used for both hadrons and ions, together with the ABLA model for deexcitation. For the energy below $50 \mathrm{AMeV}$ the inelastic interaction was modeled using the precompound exciton model. The interactions of neutrons with the energy below $20 \mathrm{MeV}$ are modeled using the high precision experimental neutron database from ENDF/B VII-1 library.

\section{Results and discussion}

The spatial distribution of accumulated isotopes depends on the projectile type, initial energy and the threshold energy for the isotopes. The comparison between the radial distribution of ${ }^{59} \mathrm{Fe},{ }^{58} \mathrm{Co}$ and ${ }^{48} \mathrm{~V}$ in the case of irradiation by $0.66 \mathrm{GeV}$ protons and $4 \mathrm{AGeV}$ deuterons is shown in figure 3 . The accumulation rates of the isotopes $N_{\text {isot }}$ are plotted as functions of radius, for $\mathrm{z}=39 \mathrm{~cm}$ (after the second section, figure $3 \mathrm{a}$ ) and $\mathrm{z}=78 \mathrm{~cm}$ (after the fifth section, figure $3 \mathrm{~b}$ ). The data are scaled by the factors shown in the figure, in order to present all three isotopes in the same figure.

The distribution of an isotope reflects the distribution of particles with the energy above the isotope threshold energy. The distributions can be well parameterized with one (for ${ }^{60} \mathrm{Co},{ }^{59} \mathrm{Fe}$ ) or two exponentials (for the isotopes with higher energy threshold). The corresponding fits are shown in figure 3. Due to the fit simplicity, we choose it to present the distributions for calculating the values of isotopes at a given distance from the beam axis.

The comparison between the accumulation rates of isotopes under irradiation by proton $0.66 \mathrm{GeV}$, deuteron $4 \mathrm{AGeV}$ and carbon $4 \mathrm{AGeV}$ beams is given in table 1 . In the first column of table 1 the longitudinal ( $\mathrm{z}$ ) and radial (r) positions of the samples are given. The second column contains infor- 

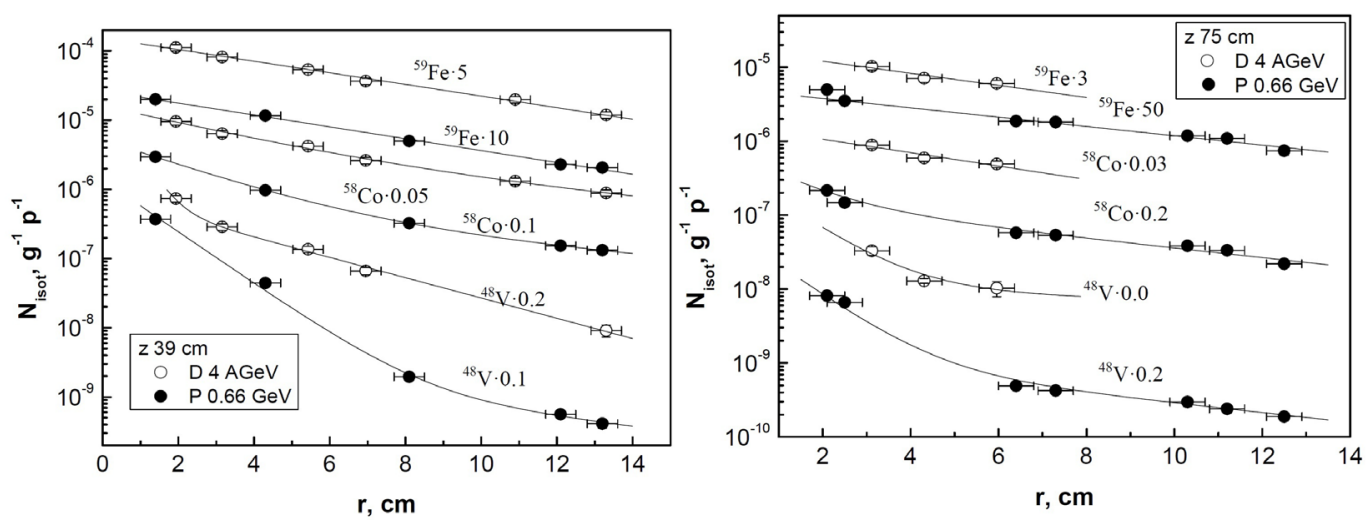

Figure 3. Radial distributions of ${ }^{59} \mathrm{Fe},{ }^{58} \mathrm{Co},{ }^{48} \mathrm{~V}$ at (a) $\mathrm{z}=39 \mathrm{~cm}$ and (b) $\mathrm{z}=78 \mathrm{~cm}$ under irradiation by $0.66 \mathrm{GeV}$ protons and $4 \mathrm{AGeV}$ deuterons. Lines show the exponential fit (see the text).

mation on the projectile and its energy per nucleon. The experimental and simulated using Geant 4 code values for the accumulation rates of the isotopes are given in the remaining columns.

Let us analyze the change of the particle spectrum at a given position, under irradiation of the target with proton $0.66 \mathrm{GeV}$, deuteron $4 \mathrm{AGeV}$, and carbon $4 \mathrm{AGeV}$ beams. The coefficient $R_{i, j}$ defined as the ratio of the accumulation rate for the isotope $i$ and the accumulation rate for the isotope with the lowest energy threshold (in our case ${ }^{60} \mathrm{Co}$ ) under the target irradiation by the beam $\mathrm{j}$ is used as a measure of the spectrum hardness:

$$
R_{i, j}=N_{i, j} / N_{60} \mathrm{Co}, j
$$

The values of $R_{i, j}$ are determined with higher precision than the accumulation rate $N_{i, j}$ because the errors for $R_{i, j}$ do not include the errors from the beam intensity measurements. The ratios $R_{i, D 4 A G e V} / R_{i, P 0.66 G e V}$ as functions of the isotope threshold energy are shown in figure 4a for different positions inside the target. It can be seen that the hardness of the particle spectra under irradiation by $4 \mathrm{AGeV}$ deuterons increases with increasing radial and longitudinal distance inside the target. The growth is more pronounced for particles with the energy above $70 \mathrm{MeV}$. After the first $10 \mathrm{~cm}$ (after section $2-\mathrm{z}=39 \mathrm{~cm}$ ) the ratio $R_{i, D 4 A G e V} / R_{i, P 0.66 G e V}$ for ${ }^{48} \mathrm{~V}$ is 1 at a radius of $6 \mathrm{~cm}$, and increases to 1.3 at a radius of $13.9 \mathrm{~cm}$. After the fifth section $(40 \mathrm{~cm}$ in uranium) the ratio reaches a value of 6 . These values reflect the generation of secondaries with higher energy, and with a more pronounced forward peak during the irradiation by the deuteron beam with an energy of $4 \mathrm{AGeV}$ in comparison with the $0.66 \mathrm{GeV}$ proton beam.

An important conclusion made from the comparison between spectra obtained under the target irradiation by carbon and deuteron beams with the same energy per nucleon (4 AGeV) illustrated in figure $4 \mathrm{~b}$ is that in all positions the spectra obtained with the carbon beam are harder than the spectra obtained with the deuteron beam and the difference increase with increasing threshold energy of the isotope. The ratio $R_{i, C 4 A G e V} / R_{i, D 4 A G e V}$ for ${ }^{48} \mathrm{~V}$ is 1.4 at the second section and a radius of 6 $\mathrm{cm}$, and 1.55 at the fifth section. The simulation poorly reproduces the hardening of the spectrum. The simulation with the BC-Bert model reproduces the trend, but significantly underestimates the effect. The simulation with the INCL model predicts the spectra with similar hardness for carbon and deuteron beams. 
Table 1. Experimental and simulated accumulation rates of the isotopes, under irradiation by proton $0.66 \mathrm{GeV}$, deuteron $4 \mathrm{AGeV}$ and carbon $4 \mathrm{AGeV}$ beams.

\begin{tabular}{|c|c|c|c|c|c|c|c|c|}
\hline $\begin{array}{l}\mathrm{z} / \mathrm{R}, \\
\mathrm{cm}\end{array}$ & $\begin{array}{l}\text { Part/ } \\
\text { Energy }\end{array}$ & $\begin{array}{l}\text { Exp/ } \\
\text { Sim }\end{array}$ & $\begin{array}{l}{ }^{60} \mathrm{Co} \\
g^{-1} p^{-1}\end{array}$ & $\begin{array}{l}{ }^{59} \mathrm{Fe} \\
g^{-1} p^{-1}\end{array}$ & $\begin{array}{l}{ }^{58} \mathrm{Co} \\
g^{-1} p^{-1}\end{array}$ & $\begin{array}{l}{ }^{57} \mathrm{Co} \\
g^{-1} p^{-1}\end{array}$ & $\begin{array}{l}{ }^{56} \mathrm{Co} \\
g^{-1} p^{-1}\end{array}$ & $\begin{array}{l}{ }^{48} \mathrm{~V} \\
g^{-1} p^{-1}\end{array}$ \\
\hline \multirow[t]{9}{*}{$39 / 6.1$} & \multirow[t]{3}{*}{$\mathrm{P} / 0.66$} & $\exp$ & $(8.1 \pm 0.8) \mathrm{e}-6$ & $(7.9 \pm 0.8) \mathrm{e}-7$ & $(5.5 \pm 0.6) \mathrm{e}-6$ & $(1.8 \pm 0.2) \mathrm{e}-6$ & $(2.7 \pm 0.3) \mathrm{e}-7$ & $(8.6 \pm 0.9) \mathrm{e}-8$ \\
\hline & & BC-Bert & $9 e-6$ & $1 \mathrm{e}-6$ & $6 e-6$ & $1.7 e-6$ & $2.7 \mathrm{e}-7$ & $6.7 \mathrm{e}-8$ \\
\hline & & INCL & $8.2 \mathrm{e}-6$ & $1 e-6$ & $5.55 e-6$ & $1.7 \mathrm{e}-6$ & $2.65 e-7$ & $9 e-8$ \\
\hline & \multirow[t]{3}{*}{ D 4} & $\exp$ & $(1.1 \pm 0.1) \mathrm{e}-4$ & $(9.1 \pm 1) \mathrm{e}-6$ & $(6.7 \pm 0.7) \mathrm{e}-5$ & $(1.9 \pm 0.2) \mathrm{e}-5$ & $(2.5 \pm 0.3) \mathrm{e}-6$ & $(9.5 \pm 1) e-7$ \\
\hline & & BC-Bert & $1.14 \mathrm{e}-4$ & $9.5 e-6$ & $6.7 e-5$ & $1.9 e-5$ & $2.46 \mathrm{e}-6$ & $9.3 e-7$ \\
\hline & & INCL & $8.1 \mathrm{e}-5$ & $1 e-5$ & $5.9 e-5$ & $1.5 e-5$ & $2.1 \mathrm{e}-6$ & $5.9 \mathrm{e}-7$ \\
\hline & \multirow[t]{3}{*}{$\mathrm{C} / 4$} & $\exp$ & $(5.5 \pm 0.6) \mathrm{e}-4$ & $(4.8 \pm 0.5) \mathrm{e}-5$ & $(3.9 \pm 0.4) \mathrm{e}-4$ & $(1.2 \pm 0.13) \mathrm{e}-4$ & $(1.8 \pm 0.2) \mathrm{e}-5$ & 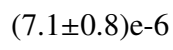 \\
\hline & & BC-Bert & $5.5 e-4$ & $7 e-5$ & $4.7 \mathrm{e}-4$ & $1.15 \mathrm{e}-4$ & $1.6 e-5$ & $3.56 \mathrm{e}-6$ \\
\hline & & INCL & $4.3 \mathrm{e}-4$ & $5.5 e-5$ & $3.3 \mathrm{e}-4$ & $8.5 e-5$ & $1.25 \mathrm{e}-5$ & $3.9 \mathrm{e}-6$ \\
\hline \multirow[t]{9}{*}{$39 / 13.9$} & \multirow[t]{3}{*}{$\mathrm{P} / 0.66$} & $\exp$ & $(4.3 \pm 0.4) \mathrm{e}-6$ & $(1.7 \pm 0.2) \mathrm{e}-7$ & $(1.2 \pm 0.1) \mathrm{e}-6$ & $(3.5 \pm 0.35) \mathrm{e}-7$ & $(4.3 \pm 0.4) \mathrm{e}-8$ & $(3.9 \pm 0.4) \mathrm{e}-9$ \\
\hline & & BC-Bert & $3.4 \mathrm{e}-6$ & $1.9 \mathrm{e}-7$ & $1.2 \mathrm{e}-6$ & $2.9 \mathrm{e}-7$ & $3.6 \mathrm{e}-8$ & $3.1 \mathrm{e}-9$ \\
\hline & & INCL & $3.2 \mathrm{e}-6$ & $1.8 \mathrm{e}-7$ & $1.1 \mathrm{e}-6$ & $3 e-7$ & $3.6 e-8$ & $3.2 \mathrm{e}-9$ \\
\hline & \multirow[t]{3}{*}{$\mathrm{D} / 4$} & $\exp$ & $(6 \pm 0.6) e-5$ & $(2.1 \pm 0.2) \mathrm{e}-6$ & $(1.64 \pm 0.17) \mathrm{e}-5$ & $(4.9 \pm 0.5) \mathrm{e}-6$ & $(6.2 \pm 0.7) \mathrm{e}-7$ & $(7.2 \pm 0.8) \mathrm{e}-8$ \\
\hline & & BC-Bert & $4.36 \mathrm{e}-5$ & $2.76 e-6$ & $1.7 \mathrm{e}-5$ & $4.2 \mathrm{e}-6$ & $5.1 \mathrm{e}-7$ & $5.4 \mathrm{e}-8$ \\
\hline & & INCL & $3.3 e-5$ & $2.2 \mathrm{e}-6$ & $1.25 \mathrm{e}-5$ & $3 e-6$ & $3.8 \mathrm{e}-7$ & $5.9 \mathrm{e}-8$ \\
\hline & \multirow[t]{3}{*}{$\mathrm{C} / 4$} & $\exp$ & $(2.7 \pm 0.3) \mathrm{e}-4$ & $(1.1 \pm 0.1) \mathrm{e}-5$ & $(8.6 \pm 0.9) \mathrm{e}-5$ & $(2.6 \pm 0.3) \mathrm{e}-5$ & $(3.7 \pm 0.5) \mathrm{e}-6$ & - \\
\hline & & BC-Bert & $2.54 \mathrm{e}-4$ & $1.45 \mathrm{e}-5$ & $8.64 e-5$ & $2 \mathrm{e}-5$ & $2.4 \mathrm{e}-6$ & \\
\hline & & INCL & $1.77 \mathrm{e}-4$ & $1.23 e-5$ & $8.2 \mathrm{e}-5$ & $1.75 e-5$ & $2.2 \mathrm{e}-6$ & \\
\hline \multirow[t]{9}{*}{$65 / 6.5$} & \multirow[t]{3}{*}{$\mathrm{P} / 0.66$} & $\exp$ & $(2.9 \pm 0.3) \mathrm{e}-6$ & $(1 \pm 0.1) \mathrm{e}-7$ & $(7.8 \pm 0.8) \mathrm{e}-7$ & $(2.44 \pm 0.24) \mathrm{e}-7$ & (3.6 60.4$) \mathrm{e}-8$ & $(8.7 \pm 0.9)$ e- 9 \\
\hline & & BC-Bert & $1.9 e-6$ & $7.53 \mathrm{e}-8$ & $4.86 \mathrm{e}-7$ & $1.45 \mathrm{e}-7$ & $2.34 \mathrm{e}-8$ & $5.4 \mathrm{e}-9$ \\
\hline & & INCL & $1.7 e-6$ & $7.3 e-8$ & $4.74 \mathrm{e}-7$ & $1.54 \mathrm{e}-7$ & $2.3 e-8$ & $5.16 \mathrm{e}-9$ \\
\hline & \multirow[t]{3}{*}{$\mathrm{D} / 4$} & $\exp$ & $(6.3 \pm 0.65) \mathrm{e}-5$ & $(4.1 \pm 0.4) \mathrm{e}-6$ & $(2.9 \pm 0.3) \mathrm{e}-5$ & $(8.7 \pm 0.9) \mathrm{e}-6$ & $(1.26 \pm 0.13) \mathrm{e}-6$ & $(5 \pm 0.5) e-7$ \\
\hline & & BC-Bert & $4.8 \mathrm{e}-5$ & $4.08 \mathrm{e}-6$ & $2.68 \mathrm{e}-5$ & $7.07 e-6$ & $1.04 \mathrm{e}-6$ & $2.77 \mathrm{e}-7$ \\
\hline & & INCL & $4.65 e-5$ & $4.57 \mathrm{e}-6$ & $2.73 e-5$ & $7.24 \mathrm{e}-6$ & $1.09 \mathrm{e}-6$ & $3.64 \mathrm{e}-7$ \\
\hline & \multirow[t]{3}{*}{$\mathrm{C} / 4$} & $\exp$ & $(3.1 \pm 0.3) \mathrm{e}-4$ & $(2.3 \pm 0.2) e-5$ & $(1.7 \pm 0.2) \mathrm{e}-4$ & $(5.6 \pm 0.6) \mathrm{e}-5$ & $(8.4 \pm 1) \mathrm{e}-6$ & $(3.6 \pm 0.5) \mathrm{e}-6$ \\
\hline & & BC-Bert & $2.73 e-4$ & $2.24 \mathrm{e}-5$ & $1.49 \mathrm{e}-4$ & $3.85 e-5$ & $5.8 \mathrm{e}-6$ & $1.69 \mathrm{e}-6$ \\
\hline & & INCL & $2.5 \mathrm{e}-4$ & $2.36 e-5$ & $1.49 \mathrm{e}-4$ & $3.83 e-5$ & $5.8 \mathrm{e}-6$ & $2.02 \mathrm{e}-6$ \\
\hline \multirow[t]{9}{*}{$78 / 6.9$} & \multirow[t]{3}{*}{$\mathrm{P} / 0.66$} & $\exp$ & $(1.8 \pm 0.2) \mathrm{e}-6$ & $(3.9 \pm 0.4) \mathrm{e}-8$ & $(2.8 \pm 0.3) \mathrm{e}-7$ & $(8.95 \pm 0.9) \mathrm{e}-8$ & $(1.2 \pm 0.12) \mathrm{e}-8$ & $(2.6 \pm 0.3) \mathrm{e}-9$ \\
\hline & & BC-Bert & $6.94 \mathrm{e}-7$ & $1.84 \mathrm{e}-8$ & $1.2 \mathrm{e}-7$ & $3.73 e-8$ & $6.1 \mathrm{e}-9$ & $1.3 \mathrm{e}-9$ \\
\hline & & INCL & $6.25 \mathrm{e}-7$ & $1.8 \mathrm{e}-8$ & $1.26 \mathrm{e}-7$ & $4 e-8$ & $5.56 \mathrm{e}-9$ & $1.14 \mathrm{e}-9$ \\
\hline & \multirow[t]{3}{*}{$\mathrm{D} / 4$} & $\exp$ & $(3 \pm 0.3) e-5$ & $(2 \pm 0.2) \mathrm{e}-6$ & $(1.6 \pm 0.2) \mathrm{e}-5$ & $(5 \pm 0.5) \mathrm{e}-6$ & $(6.4 \pm 0.7) \mathrm{e}-7$ & $(2.6 \pm 0.3) \mathrm{e}-7$ \\
\hline & & BC-Bert & $2 e-5$ & $1.54 \mathrm{e}-6$ & $1.13 e-5$ & $2.83 e-6$ & $4.4 \mathrm{e}-7$ & $1.24 \mathrm{e}-7$ \\
\hline & & INCL & $2 e-5$ & $1.96 \mathrm{e}-6$ & $1.35 \mathrm{e}-5$ & $3.8 \mathrm{e}-6$ & $5.66 \mathrm{e}-7$ & $2.07 \mathrm{e}-7$ \\
\hline & \multirow[t]{3}{*}{$\mathrm{C} / 4$} & $\exp$ & $(1.4 \pm 0.14) \mathrm{e}-4$ & $(1.1 \pm 0.1) e-5$ & $(9.1 \pm 0.9) \mathrm{e}-5$ & $(3.1 \pm 0.3) \mathrm{e}-5$ & $(4.5 \pm 0.6) \mathrm{e}-6$ & $(1.9 \pm 0.2) \mathrm{e}-6$ \\
\hline & & BC-Bert & $1.06 \mathrm{e}-4$ & $8.4 \mathrm{e}-6$ & $6 e-5$ & $1.6 \mathrm{e}-5$ & $2.5 e-6$ & $7.95 \mathrm{e}-7$ \\
\hline & & INCL & $1 e-4$ & $9.7 e-6$ & $6.7 e-5$ & $1.9 \mathrm{e}-5$ & $2.83 \mathrm{e}-6$ & $1.07 \mathrm{e}-6$ \\
\hline
\end{tabular}



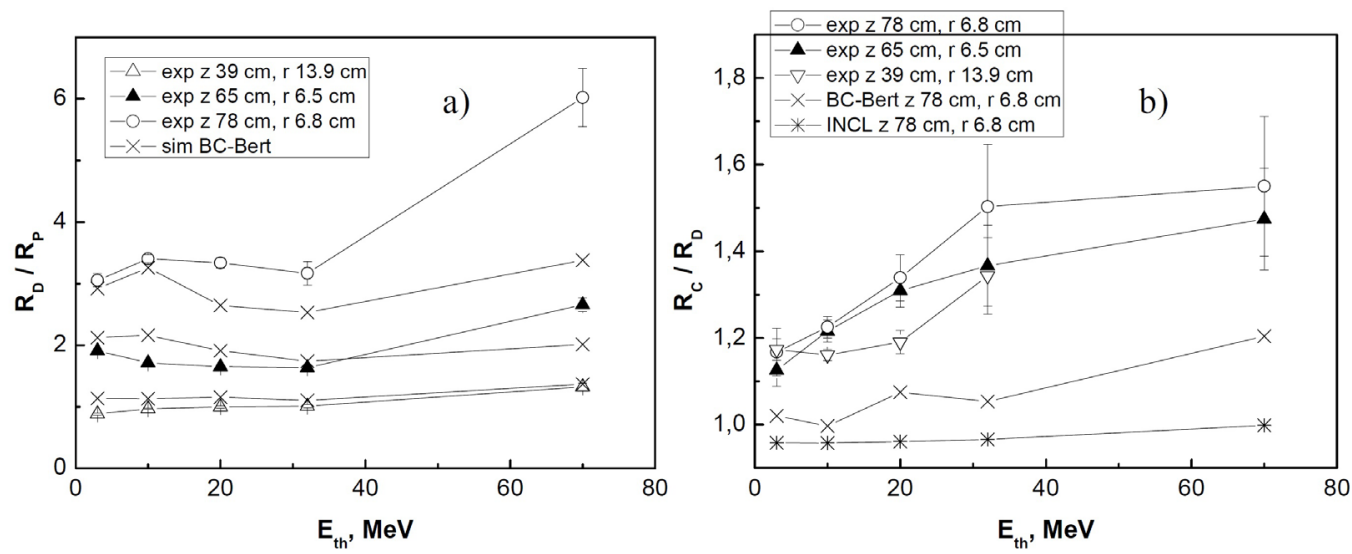

Figure 4. Experimental and simulated ratios (a) $R_{i, D 4 A G e V} / R_{i, P 0.66 \mathrm{GeV}}$ and (b) $R_{i, C 4 A G e V} / R_{i, D 4 A G e V}$ as functions of the threshold energy of the isotopes, at different positions inside the target.

\section{Conclusion}

The particle spectra and spatial distributions inside the extended uranium target "Quinta" were studied by measuring the accumulation rate of isotopes in samples of ${ }^{59} \mathrm{Co}$, under target irradiation with proton beam with energy $0.66 \mathrm{GeV}$, deuteron beam with energy $4 \mathrm{AGeV}$, and carbon beam with energy $4 \mathrm{AGeV}$. A set of 7 isotopes with threshold energy production from 0 to $70 \mathrm{MeV}$ was measured. The experimental data show that the particle spectra become harder under irradiation by carbon in comparison with deuteron beam with the same energy per nucleon.

The experimental results were compared with the simulation performed using the code Geant 4 . The simulations show good agreement with the experimental data in the proximity of the shower maximum, but underestimate the production of isotopes 1.5-2 times at the end of the target. The simulation does not reproduce correctly the hardening of the spectrum under irradiation with carbon beam in comparison with deuteron beam with the same energy per nucleon.

The obtained results demonstrate that it is necessary to improve the simulation codes and to continue the experimental investigations with the extended target irradiated by light ion beams in an energy range of $1-4 \mathrm{AGeV}$.

\section{References}

[1] R.G. Vasil'kov et al., Sov. J. At. Energy 44(4), 329-335 (1978)

[2] R.G. Alsmiller Jr. et al., Nucl. Sci. Eng. 79, 162-166 (1981)

[3] C. Rubbia et al., preprint CERN/AT/95-44 (ET) (1995)

[4] D. G. Koshkarev, Technical Physics 74, 137-139 (2004)

[5] S.R. Hashemi-Nezhad, W. Westmeier, M. Zamani-Valasiadou, B. Thomauske, R. Brandt, Ann. Nucl. Energy 38, 1144-1155 (2011)

[6] M. Paraipan, A.A. Baldin, M.G. Kadykov, S.I. Tyutyunikov, XXI Baldin ISHEPP Dubna, Russia, September 10-15 (2012)

[7] S. Agostinelli et al., Nucl. Instr. Meth. A 506, 250- 303 (2003)

[8] A.A. Baldin et al., Phys. Part. Nucl. Lett. 13, 210- 217 (2016) 